\title{
Infrared spectroscopy of the Seyfert 2 galaxy Mrk 1210
}

\author{
Ximena Mazzalay ${ }^{1}$ and Alberto Rodríguez-Ardila ${ }^{2}$ \\ ${ }^{1}$ Observatorio Astronómico de Córdoba, Laprida 854, Córdoba, Argentina \\ email: ximena@oac.uncor.edu \\ ${ }^{2}$ LNA/MCT, Rua dos Estados Unidos 154, Itajubá, MG, Brazil email: aardila@lna.br
}

\begin{abstract}
Mrk 1210 is an Sa galaxy at $z=0.01350$. Its optical spectrum displays a strong featureless continuum and Wolf-Rayet features within the central 200 pc (Storchi-Bergmann et al. 1998), evidencing the presence of a circumnuclear starburst. Here, we present SpeX nearinfrared (NIR) spectroscopy in the interval $0.8-2.4 \mu \mathrm{m}$, combined with optical $(3700 \AA-7400 \AA)$ observations, aimed at studying the physical properties of the nuclear and extended gas. Kinematics derived from the line profiles, extinction affecting the emitting gas, temperature and density indicators of the NLR, and excitation sources for the molecular and low-ionization gas are discussed. The presence of high-ionization as well as low-ionization and molecular lines in the optical and NIR spectrum suggests a strong competition between the starburst component and the ionized gas from the central engine. This makes Mrk 1210 an ideal target to study the interplay between starburst and nuclear activity in galaxies.
\end{abstract}

\section{Observational results and kinematics}

The NIR spectrum of Mrk1210 (see Figure 1) is dominated by permitted H I and He II lines, and forbidden lines of [S II], [S III] and [Fe II]. There are also present highly ionized lines of [S VIII], [S IX], and [Six], as well as numerous $\mathrm{H}_{2}$ lines. Extended [S III] and He I emission is found up to a distance of $500 \mathrm{pc}$ from the nucleus. The analysis of the emission line profiles, both allowed and forbidden, shows a narrow (FWHM $\sim 900 \mathrm{~km} / \mathrm{s}$ ) line on top of a broad (FWHM $\sim 1600 \mathrm{~km} / \mathrm{s}$ ) blue-shifted component $(\Delta \mathrm{V} \sim 120 \mathrm{~km} / \mathrm{s})$. The latter seems to be associated to a nuclear outflow, most probably located in the NLR instead of the BLR. This result rules out the presence of a hidden BLR claimed to be present in previous NIR observations of this object (Veilleux et al. 1997).

\section{Reddening}

The numerous H I and forbidden lines in the spectra of Mrk 1210 allowed us to map the intrinsic extinction affecting the NLR by means of indicators such as the Pashen decrement and the [Fe II] $1.257 \mu \mathrm{m} / 1.64 \mu \mathrm{m}$ ratio. The extinction law of Cardelli, Clayton, \& Mathis (1989) was used for this purpose.

The results reveal a dusty AGN while the extended regions are reddening free. In the inner $200 \mathrm{pc}$ we found an $E(B-V)=0.5$ from the $\mathrm{H}$ i lines ratios, except for $\mathrm{H} \alpha / \mathrm{H} \beta$ from which we found $E(B-V)=0.3$, indicating differences in dust columns. From the [Fe II] ratio $1.257 \mu \mathrm{m} / 1.64 \mu \mathrm{m}$ a large extinction value, $E(B-V)=1.5$ was derived. This supports the hypothesis that the [Fe II] lines are formed in a separate region, different from the classical NLR. At $250 \mathrm{pc}$ from the nucleus, no extinction is found from our data.

\section{Electron Density and Temperature}

Electronic densities and temperatures of the nuclear gas in Mrk 1210 were determined by means of several diagnostic line ratios. The results are summarized in Table 1. Notice 

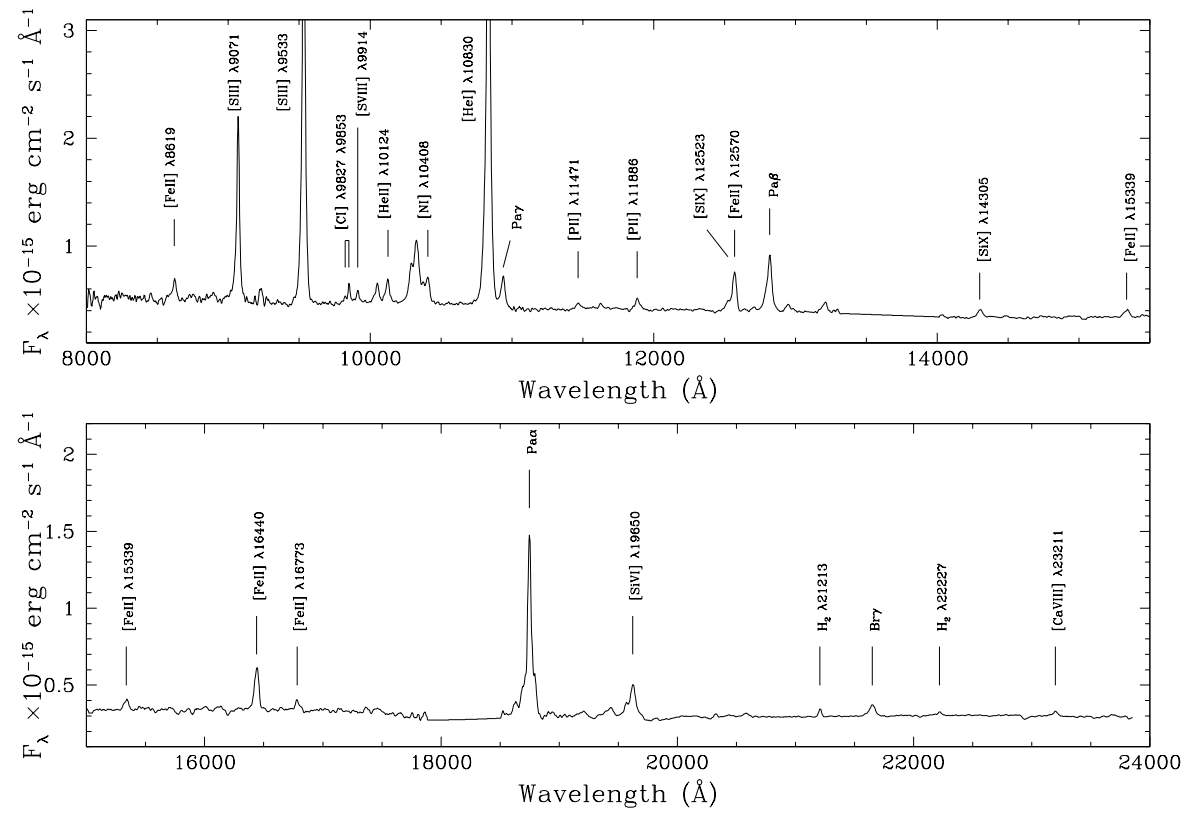

Figure 1. Observed nuclear NIR spectrum of Mrk1210 in laboratory wavelengths.

$\begin{array}{ccc}\text { Diagnostic Ratio } & N_{\mathrm{e}}\left(\mathrm{cm}^{-3}\right) & T_{\mathrm{e}}(\mathrm{K}) \\ {[\mathrm{N} \mathrm{I}](5198+5200) /(10397+10407)} & 10000 & 12000 \\ {[\mathrm{O} \text { III }](4959+5007) / 4363} & 20000 & 20000 \\ {[\mathrm{~N} \mathrm{II}](6548+6583) / 5755} & 10000 & 40000 \\ {[\mathrm{~S} \mathrm{III}](9069+9532) / 6312} & 20000 & 50000\end{array}$

Table 1. Electron densities and temperatures found for Mrk 1210

the wide range of physical conditions in the NLR gas. The large temperature derived for the $[\mathrm{S}$ III] and [N II] evidence the presence of shocks within the NLR and is consistent with the broad component and large blue asymmetry found for these lines.

\section{X-rays as a source of [Fe II] and $\mathrm{H}_{2}$ lines}

We tested if the [Fe II] and $\mathrm{H}_{2}$ emission can be attributed to X-ray heating, by calculating the emergent $\mathrm{H}_{2} 2.121 \mu \mathrm{m}$ and [Fe II] $1.644 \mu \mathrm{m}$ fluxes using the models of X-ray excitation of Maloney et al. (1996). The results show that X-rays from the AGN are responsible for nearly $30 \%$ of the molecular $\mathrm{H}_{2}$ and unable to power the [Fe II] lines. The origin of the latter emission is most probably related to the circumnuclear starburst. This hypothesis is supported by the strong extinction measured for the [Fe II] region, typical of star forming regions.

\section{References}

Cardelli, J. A., Clayton, G. C., \& Mathis, J. S. 1989, ApJ, 345, 245

Maloney, P. R., Hollenbach, D. J., \& Tielens, A. G. G. M. 1996, ApJ, 466, 561

Storchi-Bergmann, T., Fernandes, R. C., \& Schmitt, H. R. 1998, ApJ, 501, 94

Veilleux, S., Goodrich, R. W., \& Hill, G. J. 1997, ApJ, 477, 631 